\title{
Cytomegalovirus seroprevalence in pregnant women in the western region of Romania: A large-scale study
}

\author{
FLORIN GORUN $^{1 *}$, SORIN MOTOI $^{2 *}$, DANIEL MALITA $^{2}$, DAN BOGDAN NAVOLAN $^{1}$, DRAGOS NEMESCU $^{3}$, \\ TUDOR RARES OLARIU ${ }^{4}$, MARIUS CRAINA ${ }^{1}$, TATJANA VILIBIC-CAVLEK ${ }^{5,6}$, \\ IOANA CIOHAT $^{7}$, DANIEL BODA ${ }^{8,9}$ and AMADEUS DOBRESCU ${ }^{10}$ \\ Departments of ${ }^{1}$ Obstetrics and Gynecology and ${ }^{2}$ Medical Imaging and Radiology, \\ 'Victor Babes' University of Medicine and Pharmacy, 300041 Timisoara; ${ }^{3}$ Department of Obstetrics and Gynecology, \\ 'Grigore T. Popa' University of Medicine and Pharmacy, 700115 Iasi; ${ }^{4}$ Department of Parasitology, \\ 'Victor Babes' University of Medicine and Pharmacy, 300041 Timisoara, Romania; ${ }^{5}$ Department of Virology, \\ Croatian Institute of Public Health; ${ }^{6}$ School of Medicine, University of Zagreb, 10000 Zagreb, Croatia; \\ ${ }^{7}$ Antenatal Laboratory, Emergency Clinical City Hospital, 300202 Timisoara; ${ }^{8}$ Dermatology Research Laboratory, \\ 'Carol Davila' University of Medicine and Pharmacy, 050474 Bucharest; ${ }^{9}$ Department of Dermatology, \\ 'Prof. N. Paulescu' National Institute of Diabetes, Nutrition and Metabolic Diseases, 79811 Bucharest; \\ ${ }^{10}$ Departament of Surgery, 'Victor Babes' University of Medicine and Pharmacy, 300041 Timisoara, Romania
}

Received May 20, 2020; Accepted June 19, 2020

DOI: $10.3892 /$ etm.2020.8945

\begin{abstract}
Cytomegalovirus (CMV) infection is the most common congenital infection worldwide, and remains a significant cause of the neurological deficiency and sensory deafness in developed countries. Maternal primary infection, reactivation or reinfection during pregnancy may lead to fetal infection and congenital CMV syndrome. The purpose of this study was to analyze the CMV seroprevalence according to demographic features of pregnant women in western Romania as well as the evolution of CMV immunity in two time intervals. $\mathrm{IgG}$ anti-CMV antibodies were tested in sera of 8,951 pregnant women during two successive intervals: 2008-2010 ( $\mathrm{n}=1466)$ and 2015-2018 $(\mathrm{n}=7485)$. The CMV seroprevalence in women of reproductive age decreased from
\end{abstract}

Correspondence to: Dr Dan Bogdan Navolan, Department of Obstetrics and Gynecology, 'Victor Babes' University of Medicine and Pharmacy, 2 Eftimie Murgu Sq., 300041 Timisoara, Romania E-mail: navolan@yahoo.com

Dr Dragos Nemescu, Department of Obstetrics and Gynecology, 'Grigore T. Popa' University of Medicine and Pharmacy, 16 Universitatii Street, 700115 Iasi, Romania

E-mail: dnemescu@yahoo.com

${ }^{*}$ Contributed equally

Abbreviations: CMV, cytomegalovirus; HHV5, human herpes virus 5; WHO, World Health Organization; DNA, deoxyribonucleic acid

Key words: cytomegalovirus, seroprevalence, pregnant women, congenital CMV syndrome, demography
94.6 to $91.80 \%$ in the last decade. The seroprevalence was higher in women from rural areas compared with those from urban areas. These results show that the western region of Romania has a low-risk profile for primary CMV infection during pregnancy due to a large number of seropositive women. However, this risk has increased in the last ten years, from 5.4 to $8.2 \%$, which may show the need to implement a national screening program.

\section{Introduction}

Cytomegalovirus (CMV), also known as human herpesvirus-5 (HHV-5), is a double-stranded DNA virus that belongs to the Herpesviridae family. Like other herpesviruses, the CMV produces a latent infection that persists throughout the person's life after the first infection (1). The weakened immune system response may allow the reactivation of the latent virus in immunized patients (2). It can occur through iatrogenic immunosuppression (anti-rejection therapy, corticosteroid therapy) or pathologies (HIV, malignancy). Primary infection or the reactivation of a latent infection during pregnancy may lead to fetal infection and the congenital CMV syndrome. Most maternal primary infections are asymptomatic in immunocompetent women, but in some cases, the clinical picture includes symptoms similar to mononucleosis, characterized by fever, pharyngitis, lymphadenopathy, and polyarthritis (3). In the case of viral reactivation, the clinical picture is usually even more blurred.

Most CMV congenitally infected newborns are asymptomatic, with only $10 \%$ of cases being symptomatic $(4,5)$. The typical clinical symptoms in congenitally infected infants are petechiae (76\%), jaundice (67\%), hepatosplenomegaly $(60 \%)$, microcephaly (53\%), intrauterine growth retardation (50\%), chorioretinitis and optic atrophy (20\%) (6). 
CMV infection is the most common congenital infection worldwide, and remains a significant cause of the neurological deficiency and sensory deafness in developed countries $(7,8)$. The prevalence at birth varied between $0 \%$ in some studies in Egypt (1993) or Thailand (1979), and 6.2\% in the United States (1984) (4). Another report in Gambia shows a prevalence of $13.6 \%$, well above that reported in other studies; therefore, it is regarded cautiously (4). The global estimation of congenital CMV infection is $0.3-0.7 \%$ of all live births. The risk of maternal-fetal transmission is considered higher in seronegative pregnant women who acquire the primary infection during pregnancy $(4,9)$. Approximately $30 \%$ of primary infections during pregnancy led to a fetal CMV transmission, and $\sim 13 \%$ of them cause symptomatic congenital CMV syndrome (9). Therefore, populations with increased CMV seroprevalence may have lower rates of symptomatic congenital infection (9). The infection vertical transmission rate varies with gestational age at the time of the primary maternal infection: $8.8 \%$ in the pre-conceptional period, $30.6 \%$ in the first trimester, $34.1 \%$ in the 2 nd trimester and $40 \%$ in the 3 rd trimester (10).

Children with congenital infection after primary maternal infection contracted in the first trimester are more prone to sequelae of the central nervous system than the mothers who contracted the primary infection later in the pregnancy (11).

The global CMV seroprevalence among women of reproductive age is around $86 \%$, with regional variations between 70\% in Europe and 92\% in the Eastern Mediterranean region (12). Usually, the seroprevalence is higher among people with low socioeconomic status. Among women of reproductive age in European countries, the highest CMV seroprevalence is estimated to be in Turkey, with an average of $96 \%$ and the lowest in Ireland, with an average of $39 \%(12,13)$.

This study analyzed the CMV seroprevalence according to the demographic features of pregnant women in Romania as well as the evolution of CMV immunity in two intervals: 2008-2010 and 2015-2018.

\section{Patients and methods}

Study design, patients, and settings. A retrospective study was performed on 8,951 pregnant women in the western part of Romania during two successive time intervals: 2008-2010 (1,466 pregnant women tested at the Emergency Clinical City Hospital Timişoara) and 2015-2018 (7,485 pregnant women tested at SC Bioclinica srl, Timisoara, Romania). Patients were enrolled according to a consecutive-case population base. The IgG anti-CMV antibodies were determined in all pregnant women. Also, data on the year of birth, age at blood sampling, and area of residence (urban, rural) were collected and analyzed.

Serological testing. The IgG anti-CMV antibody titer was determined by the immune-chemiluminescence method using an Immulite One Machine for the interval 2008-2010, and respectively, Immulite 2000 Machine (Diagnostic Products Corporation) for the interval 2015-2018 and commercial tests (Siemens Healthcare Diagnostics Products). Depending on the cut-off values recommended by the manufacturer, the patients were classified into two categories: those with a positive titer of IgG antibodies (immunized) and those with negative or inconclusive titer (non-immunized). For statistical analysis, cases with inconclusive results were considered in combination with non-immunized cases, due to the uncertainty of immunization.

Statistical methods. The data were stored in the Astralia database and Microsoft Office Excel. For the statistical evaluation, the Instat GraphPad Prism 8.0.2 software was used. The results are presented as medians [interquartile ranges]. To assess the significance of the differences between groups Mann-Whitney U test and, respectively, Fisher's exact tests (proportions) were used. Correlations were calculated according to the Spearman rank correlation test and the corresponding P-values determined.

Ethical issues. The present study was approved by the Institutional Board of the 'Victor Babes' University of Medicine and Pharmacy (Timisoara, Romania) (approval no. 848/06.04.2011). The study meets the ethical guidelines, including adherence to the legal requirements of the study country. Informed consent was obtained from each patient.

\section{Results}

The study included 8,951 patients divided into two groups, according to the testing period: group one, 1,466 pregnant women tested between 2008-2010; and group two, 7,485 pregnant women examined between 2015-2018.

Demographic data of the study participants. Pregnant women in the first group were younger at the time of sampling as compared with the pregnant women in the second group: 28 years [6] vs. 29 years [7] years, $P<0.0001$.

The area of residence of the first group patients $(1,466)$ was urban for 1,054 (71.90\%), rural for 407 (27.76\%) and not specified for $5(0.34 \%)$ cases, while out of the $(7,485)$ women in the second group 3,570 (47.69\%) originated from urban areas, 1,930 (25.78\%) from rural areas and 1,985 (26.51\%) did not specify their origin.

Excluding the cases with not specified origin, the age analysis of the tested patients according to the residence area showed a lower percentage of young pregnant women in urban than in rural areas for both groups. This structure has reversed with increasing age, showing that pregnant women from the urban setting predominate from 26-30 years of age (Table I).

$C M V$ seroprevalence trends in the two evaluated intervals: 2008-2010 vs. 2015-2018. The CMV seropositivity of pregnant women decreased significantly from 2008-2010 to 2015-2018 from $94.68 \%(1388 / 1466)$ to $91.80 \%(6871 / 7485)(\mathrm{P}<0.0001$; $\mathrm{OR}=0.62 ; 95 \% \mathrm{CI}, 1.252-2.027)$.

The stratification of pregnant women in the first group according to the area of residence showed no significant difference for the CMV seroprevalence: urban vs. rural [94.5\% ( $\mathrm{n}=996 / 1054)$ vs. $95.33 \%(\mathrm{n}=388 / 407)(\mathrm{OR}=0.8409$; $\mathrm{P}=0.602 ; 95 \% \mathrm{CI}, 0.4966-1.423)]$, while in the second group, a significantly lower seroprevalence was found in the urban area [89.08\% ( $\mathrm{n}=3180 / 3570)$ vs. $94.92 \%(\mathrm{n}=1832 / 1930)(\mathrm{OR}=0.4362$; $\mathrm{P}<0.0001 ; 95 \%$ CI, 0.3473-0.5461)] (Table II). 
Table I. Distribution of pregnant women according to age and area of residence.

\begin{tabular}{|c|c|c|c|c|c|c|}
\hline Area of residence & $<20$ years & 21-25 years & 26-30 years & $31-35$ years & $>35$ years & Total \\
\hline $2008-2010$ & & & & & & 1,461 \\
\hline Urban & $18(1.71 \%)$ & $231(21.92 \%)$ & $483(45.83 \%)$ & $242(22.96 \%)$ & $80(7.59 \%)$ & $1,054(72.14 \%)$ \\
\hline Rural & $40(9.83 \%)$ & $129(31.70 \%)$ & $169(41.52 \%)$ & $54(13.27 \%)$ & $15(3.69 \%)$ & $407(27.85 \%)$ \\
\hline Odds ratio & 0.168 & 0.604 & 1.191 & 1.948 & 2.146 & \\
\hline P-value & $<0.0001$ & $<0.0001$ & 0.142 & $<0.0001$ & 0.006 & \\
\hline $2015-2018$ & & & & & & 5,500 \\
\hline Urban & $91(2.55 \%)$ & $526(14.73 \%)$ & $1,331(37.28 \%)$ & $1,057(29.61 \%)$ & $565(15.83 \%)$ & $3,570(64.90 \%)$ \\
\hline Rural & $185(9.59 \%)$ & $509(26.37 \%)$ & $651(33.73 \%)$ & $391(20.26 \%)$ & $194(10.05 \%)$ & $1,930(35.10 \%)$ \\
\hline Odds ratio & 0.246 & 0.482 & 1.168 & 1.664 & 1.682 & \\
\hline P-value & $<0.0001$ & $<0.0001$ & 0.008 & $<0.0001$ & $<0.0001$ & \\
\hline
\end{tabular}

Table II. Cytomegalovirus (CMV) seroprevalence according to area of residence and testing interval.

\begin{tabular}{lccc}
\hline & \multicolumn{3}{c}{ CMV IgG } \\
\cline { 2 - 4 } Testing interval & Urban & Rural & P-value \\
\hline $2008-2010$ & $94.50 \%(\mathrm{n}=996 / 1054)$ & $95.33 \%(\mathrm{n}=388 / 407)$ & 0.602 \\
$2015-2018$ & $89.08 \%(\mathrm{n}=3180 / 3570)$ & $94.92 \%(\mathrm{n}=1832 / 1930)$ & $<0.0001$ \\
P-value & $<0.0001$ & 0.803 & \\
\hline
\end{tabular}

Table III. Cytomegalovirus (CMV) seroprevalence by age groups in urban area in 2008-2010 and 2015-2018.

\begin{tabular}{|c|c|c|c|c|c|c|}
\hline \multirow[b]{2}{*}{ Age (years) } & \multicolumn{3}{|c|}{$2008-2010$} & \multicolumn{3}{|c|}{$2015-2018$} \\
\hline & Total (n) & CMV IgG (n) & CMV IgG (\%) & Total (n) & CMV IgG (n) & CMV IgG $(\%)$ \\
\hline$<20$ & 18 & 18 & 100.00 & 91 & 84 & 92.31 \\
\hline $21-25$ & 231 & 216 & 93.51 & 526 & 479 & 91.06 \\
\hline $26-30$ & 483 & 455 & 94.20 & 1,331 & 1,171 & 87.98 \\
\hline $31-35$ & 242 & 232 & 95.87 & 1,057 & 934 & 88.36 \\
\hline$>35$ & 80 & 75 & 93.75 & 565 & 512 & 90.62 \\
\hline
\end{tabular}

Table IV. Cytomegalovirus (CMV) seroprevalence by age groups in the rural area in 2008-2010 and 2015-2018.

\begin{tabular}{|c|c|c|c|c|c|c|}
\hline \multirow[b]{2}{*}{ Age (years) } & \multicolumn{3}{|c|}{$2008-2010$} & \multicolumn{3}{|c|}{$2015-2018$} \\
\hline & Total (n) & CMV IgG (n) & CMV IgG (\%) & Total (n) & CMV IgG (n) & CMV IgG (\%) \\
\hline$<20$ & 40 & 37 & 92.50 & 185 & 175 & 94.59 \\
\hline $21-25$ & 129 & 124 & 96.12 & 509 & 483 & 94.89 \\
\hline $26-30$ & 169 & 160 & 94.67 & 651 & 615 & 94.47 \\
\hline $31-35$ & 54 & 52 & 96.30 & 391 & 374 & 95.65 \\
\hline$>35$ & 15 & 15 & 100.00 & 194 & 185 & 95.36 \\
\hline
\end{tabular}

The CMV seroprevalence of the pregnant women tested in the two periods (2008-2010 vs. 2015-2018) showed a decrease in the urban area: $94.50 \%(n=996 / 1054)$ vs. $89.08 \%$ $(\mathrm{n}=3180 / 3570)(\mathrm{P}<0.0001 ; \mathrm{OR}=0.474 ; 95 \% \mathrm{CI}, 0.358-0.627)$. 
A slight decreasing trend was also observed in the rural area, but it was not significant $95.33 \%(\mathrm{n}=388 / 407)$ vs. $94.92 \%$ $(\mathrm{n}=1832 / 1930)(\mathrm{P}=0.803 ; \mathrm{OR}=0.915 ; 95 \% \mathrm{CI}, 0.547-1.486)$ (Table II).

In the urban areas there was a decreasing tendency of seroprevalence with age (Table III), but this difference was not significant: $r=-0.1780 ; 95 \% \mathrm{CI},-0.5252-0.2200 ; \mathrm{P}=0.364$ (2008-2010), and $\mathrm{r}=-0.2523 ; 95 \% \mathrm{CI},-0.5598-0.1163 ; \mathrm{P}=0.163$ (2015-2018).

We found in the rural areas an increasing, but non-significant trend, of CMV seroprevalence with age in both groups (Table IV): 2008-2010 ( $\mathrm{r}=0.3049 ; \mathrm{P}=0.157 ; 95 \% \mathrm{CI}$, -0.1355-0.6447) and, respectively, 2015-2018 ( $\mathrm{r}=0.1737$; $\mathrm{P}=0.341 ; 95 \%$ CI, $-0.1967-0.5006)$.

\section{Discussion}

Our study showed that most women aged $<20$ years are from a rural environment. The World Health Organization (WHO) estimates that 21 million women aged less than 19 years from all over the world become pregnant in developing regions, of whom 12 million give birth. According to the 2014 World Health Statistics, the global average of births in women aged $15-19$ years is $49 / 1000$ women (14). In 2014 Romania was placed second in the European Union statistics in terms of births of women aged less than 19 years, with a rate of 39.2\% (15).

In a study conducted in Romania, from 2003 to 2013, 68.72\% of 1643 teenagers who gave birth, originated from a rural environment (15). Furthermore, another study on 1,249 teenagers in the period 2010-2014, showed that the number of teenage births, in the rural environment is 3-10 times higher compared with the urban environment (16).

WHO notes that teenage pregnancies $(<19$ years) are a global problem in all countries, including those economically developed. However, they are more likely to occur in marginalized, poor communities with a low-level education system, or lack of employment opportunities. Also, young women from low socioeconomic backgrounds, who want to avoid pregnancy, cannot do so due to knowledge gaps and low availability of contraceptive methods. The WHO also notes that in some communities with low socio-economic status, women marry earlier, estimating that $\sim 39 \%$ of them marry before reaching 18 years of age in developing countries.

The CMV seroprevalence in our area in 2010 was over $94 \%$. That indicates an increased seroprevalence of CMV infection in Romania compared with that found in the developed countries from the European Union ( 40-70\%). Compared with other non-European countries, the seroprevalence of CMV infection in reproductive age women was estimated between $60-70 \%$ in Australia, while in United States the statistics indicate a prevalence of $\sim 60 \%$ (12).

In pregnant women tested from 2015 to 2018 , the CMV seroprevalence was $91.80 \%$, showing a decreasing tendency compared with the period 2008-2010, but exceeding the global average of $86 \%$ and the European average of $70 \%$. The seroprevalence in our area was comparable with the prevalence found in the Eastern Mediterranean countries, where the seroprevalence average was $92 \%$ (12). In Croatia, in the years 2005-2009, the women of reproductive age had a CMV seroprevalence of $75.3 \%$ while between the period 2013-2015 this indicator reached $78 \%(17,18)$. These studies showed an increase in seroprevalence over the years contrary to the results obtained in our study.

CMV seroprevalence assessment may be useful in establishing principles for screening and educating pregnant women. We have identified several screening guidelines for CMV during pregnancy. However, certain guidelines do not recommend routine CMV testing during pregnancy in research settings (19-21).

In this study, it was observed that the CMV seroprevalence is lower in the urban areas than in the rural areas $(\mathrm{P}=0.803$; $\mathrm{OR}=1.092$; 95\%CI, 0.6729-1.826) and the decreasing tendency during the two intervals is more pronounced in the urban environment $(\mathrm{P}<0.0001$; OR=2.106; 95\% CI, 1,593-2,789). This evolution may be due to socioeconomic conditions, better hygiene standards in the urban environment, and higher educational level compared with rural areas. Several global studies showed a difference in CMV seroprevalence from 10-30\% in people with low socioeconomic status compared with those with high socioeconomic status (13).

CMV seroprevalence is associated in several studies with maternal age. For example, seroprevalence increases with maternal age in all 32 studies reviewed by Cannon et al (13). In the present study, there was no significant association between maternal age and seroprevalence. However, we observed a lower CMV seroprevalence in the group of women aged 26-35 years tested 2015-2018 in the urban areas. Similarly, three recent studies have reported the lowest seroprevalence in women aged 30-35 years (22), 25-35 years (23), and 31-35 years (24). Some authors of these studies have speculated that an increased seroprevalence in young women can be attributed to socioeconomic status. Similar to our results, in a study from Italy on 2817 pregnant women, no significant difference in seroprevalence was found between age groups (25).

In conclusion, the CMV seroprevalence in women of reproductive age in Western Romania decreased in the last decade from 94.6 to $91.80 \%$ and was higher in women from rural areas compared with those from urban areas. These results show that Timisoara region has a low-risk profile for primary CMV infection during pregnancy due to a large number of seropositive women. However, this risk has increased in the last decade, from 5.4 to $8.2 \%$, which may show the need to implement a national screening program.

\section{Acknowledgements}

Not applicable.

\section{Funding}

No funding was received.

\section{Availability of data and materials}

The datasets used and/or analyzed during the present study are available from the corresponding author on reasonable request.

\section{Authors' contributions}

FG, DBN, DN, RTO and TVC were involved in the conception of the study and the data interpretation. SM, DM, DB, MC, 
IC and AD contributed to the data acquisition and performed statistical analysis. IC performed serological testing. FG, DBN, DN, RTO and TVC wrote the manuscript. SM, DM, $\mathrm{MC}, \mathrm{DB}$ and $\mathrm{AD}$ revised the manuscript for important intellectual content. All authors read and approved the final version of the manuscript.

\section{Ethics approval and consent to participate}

The study was approved by the Institutional Board of the 'Victor Babes' University of Medicine and Pharmacy (Timisoara, Romania) (approval no. 848/06.04.2011). The study meets the ethical guidelines, including adherence to the legal requirements of the study country. Informed consent was obtained from each patient.

\section{Patient consent for publication}

Not applicable.

\section{Competing interests}

The authors declare that they have no competing interests.

\section{References}

1. Gupta M and Shorman M: Cytomegalovirus. In: StatPearls. StatPearls Publishing, Treasure Island, FL, 2020.

2. Harrison T, Kasper D, Hauser S, Jameson J, Fauci A, Longo D and Loscalzo J: Harrison's Principles of Internal Medicine. 20th Edition. McGraw-Hill Education, New York, NY, pp1361-1365, 2018.

3. Cunningham G, Leveno K and Gilstrap L: Williams Obstetrics. 22nd edition. McGraw-Hill Professional Publishing, New York, NY, 2005.

4. Kenneson A and Cannon MJ: Review and meta-analysis of the epidemiology of congenital cytomegalovirus (CMV) infection. Rev Med Virol 17: 253-276, 2007.

5. Fowler KB and Boppana SB: Congenital cytomegalovirus infection. Semin Perinatol 42: 149-154, 2018.

6. Kagan KO and Hamprecht K: Cytomegalovirus infection in pregnancy. Arch Gynecol Obstet 296: 15-26, 2017.

7. Marsico C and Kimberlin D: Congenital cytomegalovirus infection: Advances and challenges in diagnosis, prevention and treatment. Ital J Pediatr 43: 38, 2017.

8. Mussi-Pinhata MM, Yamamoto AY, Moura Brito RM, de Lima Isaac M, de Carvalho e Oliveira PF, Boppana S and Britt WJ: Birth prevalence and natural history of congenital cytomegalovirus infection in a highly seroimmune population. Clin Infect Dis 49: 522-528, 2009.

9. Naing ZW, Scott GM, Shand A, Hamilton ST, van Zuylen WJ, Basha J, Hall B, Craig ME and Rawlinson WD: Congenital cytomegalovirus infection in pregnancy: A review of prevalence, clinical features, diagnosis and prevention. Aust NZ J Obstet Gynaecol 56: 9-18, 2016.

10. Picone O, Vauloup-Fellous C, Cordier AG, Guitton S, Senat MV, Fuchs F, Ayoubi JM, Grangeot Keros L and Benachi A: A series of 238 cytomegalovirus primary infections during pregnancy: Description and outcome. Prenat Diagn 33: 751-758, 2013.
11. Pass RF, Fowler KB, Boppana SB, Britt WJ and Stagno S: Congenital cytomegalovirus infection following first trimester maternal infection: Symptoms at birth and outcome. J Clin Virol 35: 216-220, 2006.

12. Zuhair M,Smit GS, Wallis G, Jabbar F, Smith C, Devleesschauwer B and Griffiths P: Estimation of the worldwide seroprevalence of cytomegalovirus: A systematic review and meta-analysis. Rev Med Virol 29: e2034, 2019.

13. Cannon MJ, Schmid DS and Hyde TB: Review of cytomegalovirus seroprevalence and demographic characteristics associated with infection. Rev Med Virol 20: 202-213, 2010.

14. World Health Organization: (2014). Adolescent Pregnancy. http:// www.who.int/media-centre/factsheets/fs364/en/. Accessed Feb 17, 2015.

15. Diaconescu S, Ciuhodaru T, Cazacu C, Sztankovszky LZ, Kantor C and Iorga $\mathrm{M}$ : Teenage mothers, an increasing social phenomenon in Romania. Causes, consequences and solutions. Rev Cercet Interv Soc 51: 162-175, 2015.

16. Iorga M, Socolov R and Socolov D: An 8 years analysis of pregnancies and births among teenagers in a University Hospital in North-Eastern Romania. Rev de Cercet si Interv Soc 54: 55-65, 2016.

17. Vilibic-Cavlek T, Ljubin-Sternak S, Ban M, Kolaric B, Sviben M and Mlinaric-Galinovic G: Seroprevalence of TORCH infections in women of childbearing age in Croatia. J Matern Fetal Neonatal Med 24: 280-283, 2011.

18. Vilibic-Cavlek T, Kolaric B, Beader N, Vrtar I, Tabain I and Mlinaric-Galinovic G: Seroepidemiology of cytomegalovirus infections in Croatia. Wien Klin Wochenschr 129: 129-135, 2017 (In German).

19. Vereeck S, Vereeck S and Jacquemyn Y: Screening for cytomegalovirus: An analysis of guidelines. J Pregnancy Child Health 3: 287, 2016.

20. Khalil A, Heath P, Jones C and Soe A: Ville YG on behalf of the Royal College of Obstetricians and Gynaecologists: Congenital cytomegalovirus infection: Update on treatment: Scientific impact paper No. 56. BJOG 125: e1-e11, 2018.

21. Walker SP, Palma-Dias R, Wood EM, Shekleton P and Giles ML: Cytomegalovirus in pregnancy: To screen or not to screen. BMC Pregnancy Childbirth 13: 96, 2013.

22. Taniguchi K, Watanabe N, Sato A, Jwa SC, Suzuki T, Yamanobe Y, Sago H and Kozuka K: Changes in cytomegalovirus seroprevalence in pregnant Japanese women - a 10-year single center study. J Clin Virol 59: 192-194, 2014.

23. Odland ML, Strand KM, Nordbø SA, Forsmo S, Austgulen R and Iversen AC: Changing patterns of cytomegalovirus seroprevalence among pregnant women in Norway between 1995 and 2009 examined in the Norwegian Mother and Child Cohort Study and two cohorts from Sor-Trondelag County: A cross-sectional study. BMJ Open 3: e003066, 2013.

24. Enders G, Daiminger A, Lindemann L, Knotek F, Bäder U, Exler S and Enders M: Cytomegalovirus (CMV) seroprevalence in pregnant women, bone marrow donors and adolescents in Germany, 1996-2010. Med Microbiol Immunol (Berl) 201: 303-309, 2012.

25. De Paschale M, Agrappi C, Manco MT, Paganini A and Clerici P: Incidence and risk of cytomegalovirus infection during pregnancy in an urban area of Northern Italy. Infect Dis Obstet Gynecol 2009: 206505, 2009.

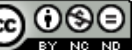

This work is licensed under a Creative Commons Attribution-NonCommercial-NoDerivatives 4.0 International (CC BY-NC-ND 4.0) License. 